\title{
European guidance for the diagnosis and management of osteoporosis in postmenopausal women
}

\author{
J. A. Kanis • N. Burlet • C. Cooper • P. D. Delmas • \\ J.-Y. Reginster • F. Borgstrom • R. Rizzoli • \\ on behalf of the European Society for Clinical \\ and Economic Aspects of Osteoporosis and Osteoarthritis \\ (ESCEO)
}

Published online: 8 March 2008

(C) International Osteoporosis Foundation and National Osteoporosis Foundation 2008

\section{Erratum to: Osteoporosis Int}

DOI 10.1007/s00198-008-0560-z

In the last row of Table 7, the fracture incidence data for the placebo and drug groups were the wrong way around. The correct rates are $2.5 \%$ for the placebo group and $1.4 \%$ for the zoledronic acid group. The corrected table is reproduced here.
The online version of the original article can be found at: http://dx.doi. org/10.1007/s00198-008-0560-z.

\section{J. A. Kanis $(\bowtie)$}

WHO Collaborating Centre for Metabolic Bone Diseases,

University of Sheffield Medical School,

Beech Hill Road,

Sheffield S10 2RX, UK

e-mail: w.j.Pontefract@shef.ac.uk

N. Burlet

IOF (International Osteoporosis Foundation),

Nyon, Switzerland

C. Cooper

MRC Epidemiology Resource Centre,

University of Southampton,

Southampton, UK

P. D. Delmas

University of Lyon and INSERM Research Unit 831,

Lyon, France
J.-Y. Reginster

WHO Collaborating Center for Public Health Aspect of Rheumatic Diseases, University of Liège,

CHU Centre Ville,

Liège, Belgium

F. Borgstrom

i3 innovus,

Stockholm, Sweden

R. Rizzoli

Service of Bone Diseases (WHO Collaborating Center for Osteoporosis Prevention) Geneva University Hospitals, Geneva, Switzerland 
Table 7 Study details and antifracture efficacy (relative risk [RR] and 95\% confidence intervals [CI]) of the major pharmacological treatments used for postmenopausal osteoporosis when given with calcium and vitamin D, as derived from randomized controlled trials

\begin{tabular}{|c|c|c|c|c|c|c|c|}
\hline \multirow[t]{2}{*}{ Intervention } & \multirow[t]{2}{*}{ Study } & \multirow[t]{2}{*}{ Entry criteria } & \multirow[t]{2}{*}{$\begin{array}{l}\text { Mean } \\
\text { age } \\
\text { (years) }\end{array}$} & \multirow[t]{2}{*}{$\begin{array}{l}\text { Number } \\
\text { of patients } \\
\text { randomised }\end{array}$} & \multicolumn{2}{|c|}{$\begin{array}{l}\text { Fracture } \\
\text { incidence } \\
\text { (percentage }^{\text {over } 3 \text { years) }}{ }^{\mathrm{d}}\end{array}$} & \multirow[t]{2}{*}{$\mathrm{RR}(95 \% \mathrm{CI})$} \\
\hline & & & & & Placebo & Drug & \\
\hline \multicolumn{8}{|c|}{ Vertebral fracture (high-risk population) } \\
\hline Alendronate $5-10 \mathrm{mg}$ & {$[51]$} & Vertebral fractures, $\mathrm{BMD} \leq 0.68 \mathrm{~g} / \mathrm{m}^{2}$ & 71 & 2,027 & 15.0 & 8.0 & $0.53(0.41-0.68)$ \\
\hline Risedronate $5 \mathrm{mg}$ & {$[55]$} & $\begin{array}{l}2 \text { vertebral fractures or } 1 \text { vertebral } \\
\text { fracture and T-score } \leq-2.0\end{array}$ & 69 & 2,458 & 16.3 & 11.3 & $0.59(0.43-0.82)$ \\
\hline Risedronate $5 \mathrm{mg}$ & {$[56]$} & $\begin{array}{l}2 \text { or more vertebral fractures-no } \\
\text { BMD entry criteria }\end{array}$ & 71 & 1,226 & 29.0 & 18.0 & $0.51(0.36-0.73)$ \\
\hline Raloxifene $60 \mathrm{mg}$ & {$[45]$} & $\begin{array}{l}\text { Vertebral fractures-no BMD } \\
\text { entry criteria }\end{array}$ & 66 & 7,705 & 21.2 & 14.7 & $0.70(0.60-0.90)$ \\
\hline Teriparatide $20 \mu \mathrm{g}^{\mathrm{a}}$ & {$[65]$} & $\begin{array}{l}\text { Vertebral fractures and FN or LS } \\
\text { T-score } \leq-1 \text { if less than } 2 \text { moderate } \\
\text { fractures }\end{array}$ & 69 & 1,637 & 14.0 & 5.0 & $0.35(0.22-0.55)$ \\
\hline Ibandronate $2.5 \mathrm{mg}$ & {$[58]$} & $\begin{array}{l}\text { Vertebral fractures and LS }-5< \\
\text { T-score } \leq-2.0\end{array}$ & 69 & 2,946 & 9.6 & 4.7 & $0.38(0.25-0.59)$ \\
\hline Ibandronate $20 \mathrm{mg}$ & {$[59]$} & $\begin{array}{l}\text { Vertebral fractures and LS }-5< \\
\text { T-score } \leq-2.0\end{array}$ & 70 & 708 & 9.6 & 4.9 & $0.50(0.34-0.74)$ \\
\hline Strontium ranelate $2 \mathrm{~g}$ & {$[68]$} & $\begin{array}{l}\text { Vertebral fractures, LS BMD } \leq \\
0.840 \mathrm{~g} / \mathrm{m}^{2}\end{array}$ & 69 & 1,649 & 32.8 & 20.9 & $0.59(0.48-0.73)$ \\
\hline Zoledronic acid $5 \mathrm{mg}$ & {$[63]$} & $\begin{array}{l}\text { FN T score } \leq-2.5, \pm \text { vertebral fracture, } \\
\text { or T-score } \leq-1.5 \text { and } 2+\text { mild or } 1 \\
\text { moderate vertebral fracture }\end{array}$ & 73 & 7,765 & 10.9 & 3.3 & $0.30(0.24-0.38)$ \\
\hline \multicolumn{8}{|c|}{ Vertebral fracture (low-risk population) } \\
\hline Alendronate $5-10 \mathrm{mg}^{\mathrm{b}}$ & {$[54]$} & FN T-score $\leq-2$ & 68 & 4,432 & 3.8 & 2.1 & $0.56(0.39-0.80)$ \\
\hline Alendronate $5-10 \mathrm{mg}^{\mathrm{b}}$ & {$[54]$} & Subgroup of women, T-score $<2.5$ & NA & 1,631 & 4.0 & 2.0 & $0.50(0.31-0.82)$ \\
\hline Raloxifene $60 \mathrm{mg}$ & {$[45]$} & $\begin{array}{l}\text { FN or LS T-score } \leq-2.5, \pm \\
\text { vertebral fractures }\end{array}$ & 66 & 7,705 & 4.5 & 2.3 & $0.50(0.40-0.80)$ \\
\hline \multicolumn{8}{|l|}{ Hip fracture } \\
\hline Alendronate $5-10 \mathrm{mg}$ & {$[51]$} & $\begin{array}{l}\text { Vertebral fractures with } \\
\mathrm{BMD} \leq 0.68 \mathrm{~g} / \mathrm{m}^{2}\end{array}$ & 71 & 2,027 & 2.2 & 1.1 & $0.49(0.23-0.99)$ \\
\hline Alendronate $5-10 \mathrm{mg}^{\mathrm{b}}$ & {$[54]$} & FN T-score $\leq-2^{c}$ & 68 & 4,432 & 0.8 & 0.7 & $0.79(0.43-1.44)$ \\
\hline Alendronate $5-10 \mathrm{mg}^{\mathrm{b}}$ & {$[54]$} & $\begin{array}{l}\text { FN T-score } \leq-2.5^{\mathrm{c}} \\
(\text { subgroup analysis })\end{array}$ & NA & 1,631 & 1.6 & 0.7 & $0.44(0.18-1.97)$ \\
\hline $\begin{array}{l}\text { Risedronate } 2.5 \\
\text { and } 5 \mathrm{mg}\end{array}$ & {$[57]$} & $\begin{array}{l}\text { T-score }<-3^{\mathrm{c}} \text { or }<-2^{\mathrm{c}} \text { and } \geq 1 \\
\text { non-skeletal risk factor for hip } \\
\text { fracture (subgroup analysis osteoporotic } \\
\text { patients } 70-79 \text { years) }\end{array}$ & 77 & 9,331 & 3.2 & 1.9 & $0.60(0.40-0.90)$ \\
\hline $\begin{array}{l}\text { Raloxifene } 60 \text { and } \\
120 \mathrm{mg}\end{array}$ & {$[45]$} & $\begin{array}{l}\text { FNorLST-score } \leq-2.5, \pm \\
\text { vertebral fractures }\end{array}$ & 66 & 7,705 & 0.7 & 0.8 & $1.10(0.60-1.90)$ \\
\hline Strontium ranelate $2 \mathrm{~g}$ & {$[69]$} & $\begin{array}{l}\text { Osteoporosis }(\mathrm{T} \text {-score }<-2.5 \text { ) with } \\
\text { or without prior fracture }\end{array}$ & 77 & 4,932 & 3.4 & 2.9 & $0.85(0.61-1.19)$ \\
\hline Strontium ranelate $2 \mathrm{~g}$ & {$[69]$} & $\begin{array}{l}\text { Age } \geq 74 \text { with T-score } \leq-2.4^{\mathrm{c}} \\
\quad \text { (subgroup analysis) }\end{array}$ & 80 & 1,977 & 6.4 & 4.3 & $\begin{array}{l}0.64(0.412- \\
0.997)\end{array}$ \\
\hline Zoledronic acid $5 \mathrm{mg}$ & {$[63]$} & $\begin{array}{l}\text { FN T score } \leq-2.5 \text { or less, } \pm \text { vertebral } \\
\text { fracture, or T-score } \leq-1.5 \text { and } 2+ \\
\text { mild or } 1 \text { moderate vertebral fracture }\end{array}$ & 73 & 7,765 & 2.5 & 1.4 & $0.59(0.42-0.83)$ \\
\hline
\end{tabular}

FN: femoral neck; LS: lumbar spine: NA: not available

a 20 -month study

b 4.2 -year study

${ }^{\mathrm{c}} \mathrm{BMD}$ adjusted to NHANES population

${ }^{\mathrm{d}}$ Except where indicated in column 1 